\title{
Editorial \\ Dynamic long distance management of patient variables: Application in ameliorating chemotherapy-associated side effects
}

\section{Background}

The N ovember 2007 issue of Annals of Oncogy carries a very interesting article on the use of mobile phone technology for managing chemotherapy-associated side effects. ${ }^{[1]}$ The authors report on the use of a "novel" technology based on automatic responses from the server as well as feedback reinforcement from a nurse to assure the patients about its appropriateness.

This initiative was based on the UK government directive on increasing self-responsibility and selfmanagement in health and illness - empowering the general public as well as the patient to play a proactive role to receive optimal advice and management. ${ }^{[2]}$

This development was spearheaded by a team consisting of oncologists, engineers, nurses, statistician, clinical trial staff, and patient representative. It was rolled out as a clinical study at Oxford U niversity. Patients with colon cancer and able to read, write, and comprehend English were enrolled in this study. Obviously, they also had to be able to use their cell phone (to input their symptoms twice a day).

\section{Technology}

The cell phone used required to be preloaded with a software that was developed by the team. Incidentally, the instrument used in this study was the Motorola V 600 - which is easily available in India. Patients were also provided with a digital tympanic thermometer, written manual of instructions as well as a formal training imparted by a research nurse. On the basis of the Common Terminology Criteria for Adverse Events (CTCAE), the software displayed the grading of specific symptoms/side effects that included temperature (fever), nausea, vomiting, mucositis, diarrhea, and hand foot syndrome.

Symptom data were entered via the mobile phone from home by the patient and transmitted to a central server using a secure general packet radio service (GPR S) connection. This data were displayed on the patients' phone (for him to confirm that the data entered were error-free) and on a webpage that could be accessed by the research nurse. The software developed automatically generated amber and red flags for any value that was out of range (predetermined by the team). There were 7 amber items (warnings) and 15 red parameters (urgent warnings). The research nurse (or her cover) was expected to respond within 90 min to any red alerts.

\section{Outcome Reported}

In a limited study restricted to six patients over two cycles of chemotherapy each, there was very good compliance $(98 \%)$. There were a few glitches in the automatic system as well as the process of timely response from the research nurse - but these were only to be expected, during the learning curve.

The authors state that this is a groundbreaking study. The discussion is all rosy - stating that the technology is simple (age no bar), the side effects were very few (certainly not due to this study or technology), patients had no problem entering data (probably because they were not very sick), technical glitches were quickly rectified (not specified how and in what time frame), and patients felt secure that their symptoms were being closely monitored and were proactively contacted by a nurse (feel good factor since they perceived themselves as "less bothersome" patients).

\section{Critical Review}

$U$ se of distance communication for patient management is not new. It has been in use since at least 1989. ${ }^{[3]}$ Even prior to that, there has been an attempt to replace several aspects of doctors' routine work with technology. $M$ any science friction movies show future doctors being assisted by such dramatic technology that he/she plays only a minor role in patient management.

If we take a close look at reality today, some of this is already available. Clinical trials use electronic Case R ecord Forms (eCRFs), remote capture of electronic data for centralized analysis (e.g. electrocardiograms [ECGs], digital images of computed tomography [CT] scans and magnetic resonance imaging [M RIS], $\mathrm{H}$ olter monitoring, etc.), hand-held patient diaries (e.g. personal digital assistant [PDAs] with special software) for adverse events and quality of life ( $Q \mathrm{OL})$, and even Interactive Voice R esponse Systems (ICR S) for randomization of patients. ${ }^{[4]}$ Being part of the regulatory submissions, these are validated and robust 
systems. The margin of error is minute, if at all. And the data are perfectly acceptable globally by competent regulatory and drug authorities - organizations that are truly particular regarding the ability to track, audit, and inspect the systems as well as data generated by them.

So how does management of chemotherapy side effects differ? First, its application is projected as a service rather than part of a rigid clinical trial protocol. Hence, there are a large number of variables that cannot be predicted in advance. Even if the $\mathrm{NCI}-\mathrm{CTC}$ criteria are taken as the benchmark, it is hard to imagine the "normal" having the ability and patience to go through the list and its equivalent terminology to punch in the appropriate data on their symptoms. ${ }^{[5]}$ Red flags and alerts can be incorporated into the software only for known toxicity. If the patient develops an unexpected symptom, its input will not be flagged even if it is critical. Also, the human components shall be its weakest as well as its strongest link - weakest if the doctor/nurse is not familiar with the standard operating procedures (SO Ps) and the system; strongest if the team is experienced and can put together the evidence to draw an informed conclusion beyond what is incorporated in the software.

\section{Indian Context}

Information Technology (IT) is a well-recognized strength of India. Clearly, the global reputation that India enjoys today has been spearheaded by our contribution in this field. Its benefits are spilling over to all walks of life. It may not be surprising if the software development of " $\mathrm{t}+$ M edical Server" was actually done by an Indian. The landing and take off system of H eathrow airport has been done by our countrymen, beating several global giants in this tender. If a system for management of patient side effects is to be designed, there could be no one better than an Indian IT firm.

O nce such a system is designed, tested, modified, and applicable as a service to patients, will India be able to take advantage? This is an entirely different ball game. ${ }^{[6]} \mathrm{N}$ o doubt cell phones are proliferating at an astronomical pace - making the Indian market attractive to all stakeholders in the telecommunication business. A visit to rural India showcases the reach and depth of the cell phone network - both with Bharat Sanchar Nigam Ltd. (BSNL) and the other private service providers. Current users number 28 million with 1.5 million more being added every month. ${ }^{[7]}$ Services in regional languages (including short message service [SMS]) are being offered! But the common man still communicates in the vernacular language. Any system of software that uses only English will have limited penetration and application. If we divide the population of India into three socioeconomic groups, things will be clearer. ${ }^{[8]}$ The "creamy layer" as it is popularly called, comprises the top $15 \%$ who can afford the best of treatment and are usually well educated (at least their second generation). They will prefer to go to their doctor (or even have them "flown" to their residence) in case of side effects. We do not have to worry about designing a system for them - though they can certainly avail of it in an emergency. At the bottom is the majority of our population (about $55 \%$ ) who are not sure where their next meal is going to come from. Even if they have access to cell phones, they will not be literate enough to make use of such a system. That leaves the middle class (about 30\%). They are educated, motivated, understand about their illness, and potential risks of its treatment. They are the ones who will benefit most from a system that provides rapid and automatic access to medical advice. Since this group exceeds the population of U nited States, such a service would carry immense potential.

The next question is whether it would gain acceptance by the patient. Most Indians remain uncomfortable relying on a nameless and faceless message. Leading consultants in medicine often have patients waiting for hours and even days - rather than are seen by a qualified but less well-known specialist. Another facet of the difficulty is real-time implementation of the advice provided by the system. In the rural setting, it is common for people to travel several hundred kilometers or pass through difficult terrain to reach a medical shop - leave alone a medical doctor. ${ }^{[9]}$ Under the circumstances, will the patient and his family be able to avail of the medication/remedy recommended? This shortcoming of the current system will have to be tacked simultaneously to optimize benefit. O ne alternative is to ensure that the patient goes home with sufficient supplies of "stop gap" medicine for common/ life-threatening side effects. For instance, if the patient has a significant risk of febrile neutropenia in the future, it is prudent to send him home with a couple of doses of oral broad-spectrum antibiotics. Should the complication occur and the patient cannot avail of medical help immediately, he can still take the first one or two doses of the oral antibiotics - potentially buying time to reach a medical facility while still in a condition that is salvageable.

\section{Conclusion}

Medicine is as much an art as a science - two plus two is often anything other than four. The limitation is our understanding of the biologic heterogeneity of the disease, the changing dynamics of patient-therapy 
relationship and the unpredictability variability of host factors.

Yet, the need and urge to take advantage of technology is very attractive. Since the healthcare infrastructure is already stretched beyond limits, any measure that can reduce the pressure on doctors' time is most welcome. This also has the potential to reduce costs - since human resource is often the most expensive input and prone to fatigue-related errors (the computerized system will not be susceptible to fatigue or lack of concentration). These factors alone will ensure that there is wider use of technology in the patient flow management systems. A lready GPS and hospital sensors have been able to track and utilize resources in a more efficient manner. Video phones can instantly access patientrelated information as well as enable the staff to see and talk to the patient on a real-time basis. At Artemis $\mathrm{H}$ ealth Institute, Gurgaon, remote access to H ospital Information System (HIS) and Picture Archiving and Communication System (PACS) ensure that doctors can be in touch with the latest data and reports from the comforts of his home. ${ }^{[10]}$ Voice over Internet Protocol (VolP) and virtual private network (VPN) at Amrita Institute, Kochi have already proven to save costs while providing seamless communication through computers. $\mathrm{H}$ and-held devices (like Blackberry) make this even more portable (at the cost of impinging on personal/ family time). The Government of India is promoting and making available telemedicine through satellite links. Sher-e-K ashmir Institute of M edical Sciences (SKIMS) and Gujarat Cancer Research Institute (GCRI) are examples of how this is put to optimal utilization. Tata M emorial H ospital (TMH) has also successfully providing telepathology to Barshi, a rural cancer center near Sholapur in $\mathrm{M}$ aharashatra. ${ }^{[11]}$ Some devices have already been approved or are under evaluation by US Food and Drug Administration (FDA) for clinical use. So, just like stem cells and cloning, there is no stopping the increasing application of technology in long distance patient management. The need of the hour is to ensure that adequate attention is paid to the potential pitfalls.

\section{Pros and cons of long distance management of chemotherapy-associated side effects Potential advantages}

1. immediate alert about new/worsening symptoms

2. overcoming geographical barriers

3. overcoming time barriers

4. opportunity of providing prompt medical advice

5. real-time feedback and modification of therapy

6. reduction in requirement of doctor's time

7. reduction in errors due to miscommunication/ incomplete information/human fatigue

\section{Potential disadvantages}

1. impersonal service perceived by patient

2. not applicable if patient:
a. is illiterate
b. is too sick
c. has dementia/mental compromise
d. is not familiar with technology

3. disease/therapy-specific modules will have to be developed/updated

4. technical glitches/downtime of system

5. need for continuing training of patients/new medical staff regarding the SOPS

6. medicolegal implications

7. risk of breach of patient privacy/confidentiality

8. additional measure required for secure gateway payments - where provided on fee for service basis

Parikh PM, Bhattacharyya GS

Indian Co-operative Oncology Network Academic Research Organization (ICON - ARO), Mumbai, India

Correspondence to: Dr. Parikh PM, Indian Co-operative Oncology, Network Academic Research Organization (ICON - ARO), Mumbai, India. E-mail: purvish@rediffmail.com

\section{References}

1. Weaver A, Young AM, Rowntree J, Townsend N, Pearson S, Smith J, et al. Application of mobile phone technology for managing chemotherapy associated side effects. Ann Oncol 2007; 18: 1887-92.

2. Our health, our care, our say: A new direction for community services, Dept of Health, NHS, Govt of UK. White paper accessible at http://www.dh.gov.uk/en/Healthcare/Ourhealthourcareoursay/ index.htm.

3. Lamm RD. High technology health care. Natl Forum 1989;69:14-7.

4. Nelen K. Tools for clinical trials professionals (eShowcase). Appl Clin Trials 2007; 16:32.

5. $\mathrm{NCl}$ CTC Criteria Version 2.0. Cancer Therapy Evaluation Program. DCTD, NCI, NIH, DHHS. [cited on April 30, 1999]. Available from: http://ctep.cancer.gov/forms/CTCv20_4-30-992.pdf.

6. Mony PK, Nagaraj C. Health information management: An introduction to disease classification and coding. Natl Med J India 2007;20:307-10.

7. Ahmed Z. Youth drives India's mobile phone revolution. Available from: http://news.bbc.co.uk/2/hi/business/3585257.stm.

8. Sastry PS, Parikh P. The earlier age of onset of malignancy in developing world is related to overall infection burden and could be due to the effect on telomere length. Med Hypotheses 2003;60:573-4.

9. Satpathy SK. Public health infrastructure in rural India: Challenges and opportunities. Indian J Public Health 2005;49:57-62.

10. Available from: http://www.artemishospital.in.

11. Desai S, Patil R, Kothari A, Shet T, Kane S, Borges A, et al. Static telepathology consultation service between Tata Memorial Centre, Mumbai and Nargis Dutt Memorial Charitable Hospital, Barshi, Solapur, Maharashatra: An analysis of the first 100 cases. Indian J Pathol Microbiol 2004;47:480-5. 